\title{
¿A qué refieren las afirmaciones verdaderas?
}

\author{
Ana Clara Polakof* \\ Universidad de la República, Uruguay \\ Agencia Nacional de Investigación e Innovación
}

Este trabajo parte de la referencia para intentar establecer qué es lo que hay en el mundo que permite a los seres humanos utilizar oraciones que contienen un valor de verdad e intenta demostrar que la incorporación de consideraciones lingüísticas puede hacer un aporte importante a la hora de diferenciar referencias. Para esto, se asume una estrecha relación entre el lenguaje y el mundo, así como el hecho de que es posible encontrar un vínculo entre las expresiones que el lenguaje nos permite construir y las entidades del mundo ${ }^{1}$. Esta estrecha relación nos permite partir desde el lenguaje para caracterizar las entidades que pueden ser referidas a través de la utilización de oraciones verdaderas, si es que tales entidades existen. Esto implica, por una parte, la eliminación de un problema filosófico sobre los distintos valores de verdad que nos alejaría del objetivo de intentar caracterizar las entidades antes mencionadas; y por otro, que asumimos una visión de la verdad como ser, en el sentido de que solo cuando usamos una oración verdadera podemos referir a algo ${ }^{2}$.

Para correspondencia, dirigirse a: Ana Clara Polakof (anaclarapo@gmail.com), Instituto de Lingüística, Facultad de Humanidades y Ciencias de la Educación, Magallanes 1577, 11.200 Montevideo, Uruguay.

Aunque pueda tratarse de una afirmación controvertida, en este trabajo asumimos las consecuencias que comporta su suposición.

2 El problema entre el vínculo de la verdad con la referencia o con el mundo ha sido trabajado por muchos filósofos desde los tiempos de Platón hasta la actualidad (cf. Chateaubriand 2001). 
La pregunta que da título a nuestro trabajo, que parece ser sencilla, no tiene una respuesta simple y ha sido objeto de varias inquisiciones filosóficas. Se ha planteado que las afirmaciones refieren a la verdad, siendo de esta manera la verdad un objeto, así como se ha planteado que refieren a hechos o a estados de cosas, entre otras posibles alternativas. Intentaremos dar una respuesta a esta pregunta que tenga en cuenta las respuestas que han dado algunos filósofos dentro de la filosofía analítica, así como las respuestas que se pueden dar si se intenta mediar la filosofía con la lingüística. Para lograr esto, nos acercaremos al problema, primero, desde una perspectiva filosófica - centrándonos en los desarrollos de Chateaubriand-y, luego, tendremos en cuenta cuáles son los aportes que una perspectiva lingüística podría llegar a dar al problema o a la pregunta antes formulada.

Hasta el momento, hemos hablado de oraciones, de la utilización de oraciones y de afirmaciones (a falta de un mejor término en español que evite ambigüedades) sin hacer ninguna puntualización ni diferenciación. Sin embargo, para que no haya confusiones, nos gustaría hacer las siguientes apreciaciones sobre qué queremos decir cuando utilizamos los términos antes mencionados: 1) las oraciones son estructuras gramaticales que no necesariamente tienen que tener una relación con el mundo, dado que pueden o no ser utilizadas en la comunicación; 2) en lingüística, las oraciones suelen diferenciarse de los enunciados en que solo los últimos son utilizados en la comunicación; 3) los enunciados pueden no coincidir con oraciones, es decir que si yo enuncio, por ejemplo, "sí", estoy utilizando un enunciado pero este enunciado jamás será una oración (Cf. Lyons 1997); y 4) esto nos llevó a proponer el término afirmación para referirnos a aquellos enunciados oracionales ${ }^{3}$, es decir, que coinciden en forma con oraciones como "Él quiere comer", pero que, a diferencia de estas últimas, son usados en el discurso, son emitidos por algún hablante.

Mostramos, a continuación, cuáles son los desarrollos de algunos de los filósofos que pueden acercarnos a la respuesta de la pregunta que nos hicimos anteriormente. Estos son Frege (1970), Russell (1918) y Chateaubriand (2001). Trabajamos con los dos primeros, pues son los filósofos que llevaron a Chateaubriand (2001) a hacer su propuesta, como él mismo ha planteado,

\footnotetext{
3 Debemos tener cuidado en no confundir esta noción lingüística de enunciado (el término con el que se ha traducido statement al español) con la propuesta dada por filósofos como Strawson (1976) que, creemos, se corresponden con lo que nosotros entendemos por afirmación pues se refiere al uso de las oraciones -aunque habría que agregarle la intención del hablante a la que nosotros no hemos hecho referencia ni lo haremos-(Strawson 1976).
} 
y con el propio autor pues nos centramos en sus ideas sobre los estados de cosas (Cf. Chateaubriand 2001).

Frege consideraba que si un enunciado era verdadero, su referencia debía ser lo verdadero y que si era falso su referencia debía ser lo falso. Es decir, que lo verdadero y lo falso eran objetos: "estos dos objetos son admitidos, aunque sólo sea tácitamente, por todo aquel que emita juicios, que tenga algo por verdadero, o sea, también por el escéptico" (Frege 1970: 11). A su vez, planteaba que los enunciados asertivos eran o debían ser considerados como nombres propios, idea que ha sido altamente criticada entre otros por Quine $(1960)^{4}$. Esta perspectiva nos muestra una interesante reflexión sobre la relación entre los enunciados con formas oracionales como "5 es un número primo" y aquello a lo que refieren, por lo que podría ser una vía de respuesta a la pregunta del trabajo, pero que nosotros descartamos por la dificultad que conlleva aceptar lo verdadero y la falso como objetos ${ }^{5}$.

Russell (1918) propone que en el mundo hay entidades llamadas hechos a los que se apunta mediante afirmaciones verdaderas. De esta manera, da otra solución posible a nuestra pregunta, en la que se propone una estructura de la realidad que no contiene objetos como "lo verdadero" o "lo falso". Fue finalmente este filósofo quien propuso que el mundo objetivo estaba compuesto por hechos que no eran creados por nuestro pensamiento y quien afirmó que "when I speak of a fact (...) I mean the kind of thing that makes a proposition true or false" (Russell 1918: 6). Esta realidad o mundo objetivo, que se presenta en una jerarquía ontológica, está conformada, entonces, además de por objetos y propiedades, por hechos ${ }^{6}$.

Chateaubriand (2001), por su parte, propone la existencia de estados de cosas. Si bien establece en su obra que los estados de cosas son hechos -0 , al menos, los trata como sinónimos en capítulos como el 5-, creemos que como propone una visión diferente de la relación entre las afirmaciones verdaderas y falsas con la realidad de la que planteó Russell (1918), debemos

\footnotetext{
$4 \quad$ Cuando Quine explica que una de las razones por las cuales se suele aceptar la idea de hechos es que hay "a tendency, in this case, to liken sentences to names and then posit objects for them to name", más allá de que no lo nombre y más allá de que Frege nunca llegue a hablar de hechos, claramente está haciendo alusión en parte a dicho filósofo (Quine 1960: 245).

Podría decirse que son casi tan difíciles de aceptar como objetos como "el cuadrado redondo" de Meinong (Cf. Meinong 2008).

${ }^{6} \quad$ La aceptación de los hechos como entidades en el mundo ha sido problemática y ha sido arduamente discutida por Quine quien consideraba que los hechos no eran necesarios y que era suficiente manejarse con el concepto de proposición: “(..$)$ there is no call to posit facts, certainly not over propositions (...)" (Quine 1960: 248).
} 
considerar los estados de cosas como entidades diferentes de los hechos. Diremos, entonces, que, a diferencia de Russell (1918) quien consideraba que cuando una proposición era verdadera apuntaba hacia un hecho y cuando era falsa apuntaba en contra (o hacia el otro lado) del hecho, para Chateaubriand (2001), cuando hacemos una afirmación verdadera estamos identificando un estado de cosas, así como describiendo una parte de la realidad, mientras que cuando usamos una afirmación falsa no estamos identificando un estado de cosas?

Los estados de cosas han sido definidos por Chateaubriand (2001) como combinaciones de una propiedad con un objeto y/u otras propiedades. Esto implica que, como los hechos, no pueden tener la misma jerarquía ontológica que los objetos; es decir que tienen que estar al menos un nivel por encima de los objetos, como veremos. Es posible darse cuenta, por lo antes dicho, de que la perspectiva de Chateaubriand (2001) formula la verdad como la identificación de los estados de cosas, dado que son las afirmaciones verdaderas y no las falsas las que nos permiten identificarlos. Él propone que su perspectiva posee tres ventajas: 1) le permite mantener la idea de la verdad como ser y como parte de la referencia; 2) le permite demostrar que existe un paralelismo entre el funcionamiento de las oraciones y el de las descripciones definidas; y 3 ) es compatible con "the correspondence view of truth".

Los estados de cosas se pueden caracterizar desde una perspectiva intensional y desde una perspectiva extensional. Según la primera, podemos centrarnos en su composición y, reiteremos, en que los estados de cosas son una combinación de propiedades con objetos y/u otras propiedades. Estas entidades pueden ser consideradas, desde una perspectiva ontológica, como pertenecientes a la estructura de la realidad ${ }^{9}$. Esta realidad, como ya había propuesto Frege (1970), está estructurada jerárquicamente, i.e. va a

$7 \quad$ Es, también, interesante ver más en profundidad cuál es la respuesta que Chateaubriand (2001) tiene para las afirmaciones falsas y cuál es la que tiene para las que no son verdaderas ni falsas. Sin embargo, como ninguna de ellas refiere a los estados de cosas -al menos directamente no lo hacen-, ni a entidades en el mundo, no ahondaremos en la cuestión, pero recomendamos la lectura del capítulo 1 y el 5 para entender un poco mejor estas ideas (Chateaubriand 2001).

8 Op. cit.: $57-58$.

9 Idea que no está alejada de la de Russell (1918), como mencionamos anteriormente. La diferencia radica, quizá, en la mejor definición de los estados de cosas y la eliminación de la ambigüedad que provocaba la posibilidad de hablar de hechos como si fueran los referentes de las proposiciones, desde una interpretación ontológica, o como si fueran proposiciones verdaderas, desde una interpretación proposicional (Chateaubriand 2001: 165). 
haber entidades en el mundo que tengan distintos niveles de jerarquía. Los objetos estarán en el nivel más bajo de la jerarquía (nivel 0) y los estados de cosas -debido a que son combinaciones entre propiedades y objetos u otras propiedades- estarán siempre al menos un nivel por encima de los objetos. Esta estratificación de la realidad, que no puede ser cerrada para que no haya paradojas lógicas, presenta estados de cosas en todos los niveles (a excepción del 0) y al ser estos la instanciación de una propiedad con un objeto o propiedad se encontrarán siempre en el mismo nivel de la propiedad que sea instanciada ${ }^{10}$. Según la segunda perspectiva, es posible caracterizar los estados de cosas como una secuencia finita de conjuntos, relaciones, objetos, entre otros. Podemos, entonces, diferenciar entre distintos estados de cosas: hay unos que se componen entre un conjunto y su elemento, otros en los que podemos tener una relación binaria que relaciona dos elementos, otros en los que tenemos conjuntos de conjuntos y uno de esos conjuntos, y así en más. Tenemos, entonces, estados de cosas que pueden ser identificados por oraciones como las siguientes: 1) Quine es un filósofo; 2) Frege enseñó a Carnap; y 3) Hay filósofos ${ }^{11}$. En este trabajo nos centramos en estados de cosas de estos tipos, pues nos permiten hacer un análisis de la relación entre el lenguaje y la realidad, más específicamente, de cómo podemos, a partir de las afirmaciones, referir a dicha realidad.

Hemos dicho ya que solo trabajamos con afirmaciones veradaderas lo que elimina el problema de la referencia de aquellas afirmaciones que no son verdaderas. Hemos sostenido que las afirmaciones verdaderas refieren a algo y hemos ido apuntando hacia la idea de que refieren a estados de cosas, en sintonía con Chateaubriand (2001). Hay dos conceptos que nos permiten relacionar los estados de cosas con la perspectiva lingüística, así como nos permiten discutir si efectivamente las afirmaciones refieren todas a estados de cosas o no; se trata de los conceptos de identificación y descripción. Si bien es posible establecer diferencias entre ellos -dado que, por ejemplo, es posible identificar sin describir, así como lo inverso también-, trabajamos con ambos conceptos en conjunto pues lo que a nosotros nos compete son afirmaciones que provean de una descripción de algo y, a su vez, permitan identificar ese algo. Al entrar en la identificación y la descripción debemos hacer mención a la relación que se establece entre los objetos y los estados de cosas, pues podría afirmarse que ambos presentan problemas similares cuando debemos identificarlos y describirlos.

10 La relación de diversidad, por ejemplo en $<$ Diversidad, Frege, Russell $>$, daría lugar a un estado de cosas de nivel 1 (Cf. Chateaubriand 2001: 311).

11 Chateaubriand 2001: 61. 
Se ha establecido que existe una relación ineludible cuando intentamos establecer cómo describen las afirmaciones y cómo describen los sintagmas definidos, y que la "similaridad" entre cómo describen los primeros sus estados de cosas y los segundos sus objetos es indiscutible. Sin embargo, parece claro que si para describir unos usamos oraciones y para describir otros utilizamos sintagmas, las descripciones que hacemos de ellos no son iguales. Esto se debe a y puede verse como un reflejo de que, si establecemos una jerarquía ontológica como la propuesta por Chateaubriand (2001), los estados de cosas estarán siempre al menos un nivel más arriba que los objetos y esto debe o debería reflejarse en el lenguaje ${ }^{12}$. Es posible establecer que el hecho de que los estados de cosas puedan ser juzgados y puedan tener la forma de una proposición implica una estructura más compleja que la que puede tener un sintagma que describe un objeto que, como ha afirmado Meinong (1960), no puede ser juzgado pero sí contemplado. Por lo tanto, si bien es posible hacer una analogía, como vimos, entre las descripciones de ambas entidades y su relación con la realidad, las estructuras que estas tienen van a difererir.

En la vida cotidiana recurrimos a propiedades del objeto para ayudar a identificarlo y esto podemos hacerlo mediante el uso del lenguaje. Por ejemplo, si expresamos "el perro labrador negro", utilizamos las propiedades "ser negro" y "ser labrador", para ayudarnos a identificar ese objeto contra otro objeto que pueda estar en la cercanía, como podría ser, por ejemplo, "un perro labrador blanco". Lo mismo va a suceder con los estados de cosas, pero, en este caso, debemos ser cuidadosos en separar qué es parte del estado de cosas y qué es parte de la descripción que uso para identificarlo (Cf. Chateaubriand 2001: 93 y sigs.). Cuando afirmo "el perro es un animal" o "el canino de cuatro patas es un animal", por ejemplo, estoy identificando el mismo estado de cosas, pero utilizando distintas descripciones que pueden ayudarme a identificarlo de una mejor manera, o de una manera distinta. Esto es una prueba de que los problemas a los que nos enfrentamos cuando intentamos identificar objetos o estados de cosas son similares, como bien notó Chateaubriand: "the similarity between a definite description, as a way of presenting an object, and a sentence, as a way of presenting a state of affairs, is so striking and obvious, at least for simple examples, that it just can't be missed" (Chateaubriand 2001: 76) y esa similitud se debe al hecho de que estos estados de cosas están compuestos por objetos. Por lo tanto, las

12 Siempre y cuando estemos de acuerdo con la declaración hecha al principio del trabajo en cuanto a que, al menos en parte, el lenguaje es un reflejo de la realidad. 
variaciones que podamos hacer en las afirmaciones dependerán, en parte, de las descripciones definidas que podamos utilizar.

Sin embargo, estas similitudes, que son fácilmente visualizables ${ }^{13}$, no deben permitirnos pasar por alto las diferencias estructurales entre estos complejos que hemos mencionado, pues si no son tenidas en cuenta hacen que algunas de las objeciones que presenta Quine (1960) para los hechos en Word and Object puedan ser asociadas con los estados de cosas. ¿Tiene razón Quine (1960) cuando critica la idea de que algo como "The Fifth Avenue is six miles long" 14 pueda corresponderse con un hecho, dado que para él afirmaciones como la anterior son verdaderas para la Quinta Avenida al medir efectivamente seis millas de largo? El problema, desde una perspectiva lingüística, es que cuando usamos un verbo copulativo estamos atribuyéndole al sujeto ciertas características, es decir que, de alguna manera, estamos describiendo al sujeto, en este caso la Quinta Avenida. Esto dificulta la interpretación de que una afirmación como la anterior pueda referir a algo diferente de la Quinta Avenida. Si bien es cierto que si utilizamos la oración "The Fifth Avenue is six miles long" estamos haciendo una afirmación que tiene como fin referir a algo, en este caso puntual a la extensión que posee dicha avenida, estamos a su vez haciendo referencia a una propiedad de la avenida. Entonces, si tenemos en cuenta o asumimos que los estados de cosas pueden ser combinaciones entre propiedades y objetos, y aceptamos que en la afirmación anterior estamos hablando de una propiedad del objeto al que hacemos referencia, podemos, entonces, considerar que dicha afirmación puede referir a un estado de cosas, más allá de lo convincentes que puedan parecer las objeciones de Quine (1960) ${ }^{15}$.

Hasta ahora hemos dado respuesta parcial a la pregunta inicial. Lo hemos hecho parcialmente porque existe otro problema que hemos notado con los

13 Más allá de las críticas hechas por filósofos como Quine (1960) que niegan que la relación entre una oración y el mundo pueda ser la misma que la relación entre un nombre y el mundo: "only indirection results from positing facts, in the image of sentences, as intermediaries" (Quine 1960: 246).

14 Ibídem.

15 Existen otros problemas que surgen cuando hacemos análisis lingüísticos de las oraciones que usamos para afirmar y cuando recurrimos a fenómenos lingüísticos como la nominalización en los que no entramos en detalle. Vale la pena mencionar que el hecho de que se pueda transformar una oración como Quine no es un dentista, que es uno de los ejemplos proporcionados por Chateaubriand (2001) como estado de cosas, en un sintagma determinado como el no ser Quine un dentista crea cierta paradoja al intentar diferenciar los estados de cosas de los objetos mediante la utilización de oraciones en una y la utilización de sintagmas determinados para los segundos, pero esta problemática debe aun ser resuelta e implicaría un trabajo diferente que no nos ayuda a responder la pregunta del presente trabajo. 
estados de cosas. Si tenemos en cuenta la perspectiva extensional de los estados de cosas, deberíamos considerar que todas aquellas afirmaciones en las que haya una combinación entre una propiedad y un objeto y u/otras propiedades deben referir a un estado de cosas, más allá de que pertenezcan a distintos niveles de la jerarquía ontológica. Cuando nos centramos en los estados de cosas que podrían corresponderse con el tipo 2 en los que hay una relación binaria entre dos elementos -como en Frege enseñó a Carnap ${ }^{16}$, encontramos una dificultad adicional. Hay afirmaciones, perfectamente clasificables dentro de este tipo de estados de cosas, que muestran una relación momentánea, muestran algo que nos permite identificar una entidad que es ciertamente diferente de los objetos y que suelen ser clasificadas como estados de cosas, pero que, como intentaremos demostrar, no parecen tener el mismo comportamiento que las afirmaciones como la que acabamos de nombrar. En el primer capítulo de su libro, Chateaubriand (2001) da un ejemplo de este tipo cuando se refiere a la relación "estar en" en "I am in my living room right now" (Chateaubriand 2001: 47-48). La diferencia entre estos dos tipos de afirmaciones es que, si bien ambas mantienen una relación con la realidad, la primera constituye una propiedad simple y llana de Frege, mientras que la segunda es una relación que se establece entre mi ser y el living en este momento determinado (Cf. Chateaubriand 2001: 84). Nos preguntamos, entonces, si es posible que ambas afirmaciones refieran a lo mismo.

Hay un par de factores, desde una perspectiva lingüística, que ayudan a que planteemos que existe una dificultad en reconocer que ambas refieren a lo mismo. En primer lugar, debemos tener en cuenta los tiempos verbales en los que fueron expresadas las afirmaciones y resulta evidente que la diferencia entre un pretérito y un presente ayudan al reconocimiento de esta dificultad. En segundo lugar, y esto ya no depende del tiempo verbal, hay verbos que nos permiten establecer relaciones que pueden dar lugar a afirmaciones que refieren a propiedades inherentes de los objetos que funcionan como sujetos, mientras que hay verbos que nos permiten construir oraciones que al ser afirmadas refieren a una relación entre objetos en los que no referimos a propiedades inherentes de estos, como en el caso de "I am in my living room right now". Esta dificultad se intensifica si nos movemos hacia otro tipo de afirmaciones como "El perro come una manzana" o "el auto cruza la calle", en los que difícilmente podría afirmarse que comer una manzana

\footnotetext{
16 Aquí, más allá de que no estemos frente a un verbo copulativo, la manera en la que se construye la afirmación claramente nos permite identificar una propiedad de Frege que se establece mediante la relación que podría expresarse como haberle enseñado a Carnap.
} 
o cruzar la calle puedan ser propiedades de perro y auto, respectivamente. El problema que planteamos aquí nos permite preguntarnos si estamos refiriendo a lo mismo con ellas que con afirmaciones como "Frege enseñó a Carnap", ¿tenemos siempre un estado de cosas o debemos postular una nueva entidad? Consideramos que cuando afirmarmos algo como "Frege enseñó a Quine" y algo como "el auto cruza la calle" no estamos refiriendo a entidades que puedan ser clasificadas dentro del mismo tipo de estado de cosas, al menos. Proponemos que, en el caso de afirmaciones como "el perro come una manzana", estamos refiriendo a eventos en el sentido davidsoniano como entidades particulares, individuales, que relacionan al menos dos objetos, que implican un cambio en el estado de cosa, consumen un tiempo determinado, entre otras características (Cf. Davidson 2001 [1981]). A diferencia de los estados de cosas, los eventos nos permiten explicar la relación existente entre las afirmaciones verdaderas del tipo anterior con la realidad ${ }^{17}$.

Para ir finalizando esta nota, haremos una síntesis de los planteos hechos en nuestro desarrollo. En primer lugar, creemos que es correcto postular que cuando enunciamos afirmaciones verdaderas estamos refiriendo a algo en el mundo. En segundo lugar, hemos planteado que, si bien existen similitudes entre el uso de las descripciones definidas como formas de presentar objetos y las afirmaciones como formas de presentar estados de cosas, existen diferencias estructurales que no pueden ser dejadas de lado. Esto nos llevó a plantear que es necesario reconocer dichas diferencias para no incurrir en errores. En tercer y último lugar, hemos definido que, si bien todas las afirmaciones refieren a algo, las afirmaciones del tipo "Frege enseñó a Carnap" y "El perro come una manzana" presentan diferencias sintácticosemánticas que deben establecer distintos tipos de relaciones con el mundo y, por lo tanto, deben referir a distintas entidades. Esto implica que, más allá de que las afirmaciones puedan llegar a tener una forma lingüística similar, la manera en la que estas se estructuran y las clases de palabras que las componen harán que nos permitan referir a distintas entidades en el mundo. Lo que vendría a demostrar la importancia de incorporar una consideración lingüística a la discusión filosófica sobre la referencia de las afirmaciones.

17 Esta afirmación puede resultar altamente conflictiva incluso para quienes aceptan los eventos como entidades en el mundo. Dentro de esta conflictividad se encuentra el mismo Davidson (2001 [1981]) quien ha propuesto que las oraciones no refieren y que son las descripciones definidas encabezadas por nominalizaciones las que demuestran que los eventos son referidos a través de términos singulares (el comer el perro la manzana). Sin embargo, consideramos que cuando los hablantes hacen una afirmación tienen la intención de referir y cuando afirman "el perro come una manzana" tienen la intención de referir al evento nombrado por el término singular anterior. 
A modo de conclusión nos gustaría dar una respuesta concisa a la pregunta que titula este trabajo: ¿a qué refieren las afirmaciones verdaderas? Las afirmaciones verdaderas refieren a entidades que pertenecen al mundo objetivo. Sin embargo, no siempre refieren al mismo tipo de entidades. Cuando las afirmaciones implican verdades sobre las propiedades de un objeto nos permiten identificar un estado de cosas determinado, pero cuando las afirmaciones implican la verdad sobre una situación determinada en el mundo estamos identificando otro tipo de entidades que aquí propusimos llamar eventos.

\section{REFERENCIAS BIBLIOGRÁFICAS}

Chateaubriand, Oswaldo. 2001. Logical forms. Part 1. Truth and description. Campinas: Coleção CLE.

Davidson, Donald. 2001 [1981]. Essays on Actions and Events. New York: Oxford University Press.

Frege, Gottlob. 1970. Sobre el sentido y la denotación. En Moro Simpson (comp.). Semántica filosófica: problemas y discusiones, pp. 3-28. Buenos Aires: Siglo XXI.

Lyons, John. 1997. Semántica lingüística: una introducción. Barcelona: Paidós.

Meinong, Alexious. 1960. The theory of objects. Traducción de Isaac Levi, D. B. Terrell, and Roderick Chisholm. En Roderick Chisholm (ed.). Realism and the Background of Phenomenology, pp. 76-117. Illinois: Free Press.

2008. Teoría del objeto y presentación personal. Traducción de Carola Pivetta. Buenos Aires: Miño y Dávila.

Quine,Willard. 1960. Word and Object. United States of America: The Masachusetts Institute of Technology.

Russell, Bertrand. 1918. The philosophy of logical atomism. En Bertrand Russell 2010 [1972]. The philosophy of logical atomism, pp. 1-110. London y New York: Routledge.

Strawson, Peter. 1976. Introduction to logical theory. New Dehli: B. I. Publications. 